Applied Mathematical Sciences, Vol. 9, 2015, no. 10, 459 - 463

HIKARI Ltd, www.m-hikari.com

http://dx.doi.org/10.12988/ams.2015.411975

\title{
On Pointwise Binomial Approximation for Independent Beta Binomial Random Variables
}

\author{
K. Teerapabolarn \\ Department of Mathematics, Faculty of Science \\ Burapha University, Chonburi 20131, Thailand
}

Copyright (c) $2014 \mathrm{~K}$. Teerapabolarn. This is an open access article distributed under the Creative Commons Attribution License, which permits unrestricted use, distribution, and reproduction in any medium, provided the original work is properly cited.

\begin{abstract}
Stein's method and the beta binomial $w$-functions are used to derive a non-uniform bound for the point metric between the distribution of a sum of $n$ independent beta binomial random variables, each with parameters $n_{i}, \alpha_{i}$ and $\beta_{i}$, and a binomial distribution with parameters $m=\sum_{i=1}^{n} n_{i}$ and $p=\frac{1}{m} \sum_{i=1}^{n} \frac{n_{i} \alpha_{i}}{\alpha_{i}+\beta_{i}}$. The bound is an appropriate measurement when all $\beta_{i}$ are large, with respect to all $n_{i}$ and $\alpha_{i}$.
\end{abstract}

\section{Mathematics Subject Classification: 60F05, 60G50}

Keywords: Beta binomial distribution, Binomial approximation, Stein's method, beta binomial $w$-function

\section{Introduction}

Let $X_{1}, \ldots, X_{n}$ be independently distributed beta binomial random variables, each with the probability function $p_{X_{i}}(k)=\left(\begin{array}{c}n_{i} \\ k\end{array}\right) \frac{B\left(k+\alpha_{i}, n_{i}-k+\beta_{i}\right)}{B\left(\alpha_{i}, \beta_{i}\right)}$ for $k=0, \ldots, n_{i}$, where $B$ is the complete beta function, and mean $\mu_{i}=\frac{n_{i} \alpha_{i}}{\alpha_{i}+\beta_{i}}$ and variance $\sigma_{i}^{2}=\frac{n_{i} \alpha_{i} \beta_{i}\left(n_{i}+\alpha_{i}+\beta_{i}\right)}{\left(\alpha_{i}+\beta_{i}\right)^{2}\left(1+\alpha_{i}+\beta_{i}\right)}$. Let $\mathcal{S}_{n}=\sum_{i=1}^{n} X_{i}$ and $\mathcal{B}_{m, p}$ denote the binomial random variable with parameters $m=\sum_{i=1}^{n} n_{i}$ and $p=\frac{1}{m} \sum_{i=1}^{n} \mu_{i}$. For $A \subseteq\{0, \ldots, m\}$, [4] gave a uniform bound for the distance between a beta binomial distribution with parameter $n, \alpha$ and $\beta$ and a binomial distribution with parameters $n$ and 
$p=\frac{\alpha}{\alpha+\beta}, d_{A}\left(\mathcal{S}_{n}, \mathcal{B}_{m, p}\right)=\left|P\left(\mathcal{S}_{n} \in A\right)-P\left(\mathcal{B}_{m, p} \in A\right)\right|$, as follows:

$$
d_{A}\left(\mathcal{S}_{n}, \mathcal{B}_{m, p}\right) \leq \frac{1-p^{m+1}+q^{m+1}}{(m+1) p q} \sum_{i=1}^{n}\left\{\left|p \beta_{i}-q \alpha_{i}\right|+\frac{\left(n_{i}-1\right) \beta_{i}}{\alpha_{i}+\beta_{i}+1}\right\} \frac{n_{i} \alpha_{i}}{\left(\alpha_{i}+\beta_{i}\right)^{2}}
$$

However, for $A=\left\{x_{0} ; x_{0} \in\{0, \ldots, m\}\right\}$ and $d_{x_{0}}\left(\mathcal{S}_{n}, \mathcal{B}_{m, p}\right)=\mid P\left(\mathcal{S}_{n}=x_{0}\right)-$ $P\left(\mathcal{B}_{m, p}=x_{0}\right) \mid$, the result in (1.1) becomes

$$
d_{x_{0}}\left(\mathcal{S}_{n}, \mathcal{B}_{m, p}\right) \leq \frac{1-p^{m+1}+q^{m+1}}{(m+1) p q} \sum_{i=1}^{n}\left\{\left|p \beta_{i}-q \alpha_{i}\right|+\frac{\left(n_{i}-1\right) \beta_{i}}{\alpha_{i}+\beta_{i}+1}\right\} \frac{n_{i} \alpha_{i}}{\left(\alpha_{i}+\beta_{i}\right)^{2}}
$$

for every $x_{0}$. It is observed that the bound is a uniform constant for the point metric $d_{x_{0}}\left(\mathcal{S}_{n}, \mathcal{B}_{m, p}\right)$. With this situation, a non-uniform bound with respect to $x_{0}$ is required. In this paper, we focus on deriving a non-uniform bound for this point metric.

\section{Method}

The following lemma gives the beta binomial $w$-functions, which are directly obtained from [3].

Lemma 2.1. For $1 \leq i \leq n$, let $w_{i}$ be the $w$-function associated with the beta binomial random variable $X_{i}$, then we have the following:

$$
w_{i}(k)=\frac{\left(n_{i}-k\right)\left(\alpha_{i}+k\right)}{\left(\alpha_{i}+\beta_{i}\right) \sigma_{i}^{2}}, k=0, \ldots n_{i} .
$$

The following relation is an important property for proving the result, which was stated by [2].

$$
\begin{aligned}
\operatorname{Cov}\left(\mathcal{S}_{n}, f\left(\mathcal{S}_{n}\right)\right) & =\sum_{i=1}^{n} \operatorname{Cov}\left(X_{i}, f\left(X_{i}+\sum_{j \neq i} X_{j}\right)\right) \\
& =\sum_{i=1}^{n} \sigma_{i}^{2} E\left[w_{i}\left(X_{i}\right) \Delta f\left(\mathcal{S}_{n}\right)\right],
\end{aligned}
$$

for any function $f: \mathbb{N} \cup\{0\} \rightarrow \mathbb{R}$ for which $E\left|w_{i}\left(X_{i}\right) \Delta f\left(\mathcal{S}_{n}\right)\right|<\infty$, where $\Delta f(x)=f(x+1)-f(x)$.

For Stein's method in the binomial approximation, it can be applied for every $m \in \mathbb{N}$ and $0<p=1-q<1$, for every $x_{0} \in\{0, \ldots, m\}$ and bounded realvalued function $f=f_{\left\{x_{0}\right\}}: \mathbb{N} \cup\{0\} \rightarrow \mathbb{R}$ defined as in [1], where $f(0)=f(1)$ 
and $f(x)=f(m)$ for $x \geq m$. So, Stein's equation for these conditions is as follows:

$$
P\left(\mathcal{S}_{n}-x_{0}\right)-P\left(\mathcal{B}_{m, p}=x_{0}\right)=E\left[\left(m-\mathcal{S}_{n}\right) p f\left(\mathcal{S}_{n}+1\right)-q \mathcal{S}_{n} f\left(\mathcal{S}_{n}\right)\right]
$$

For $x_{0}, x \in \mathbb{N} \cup\{0\},[5]$ showed that

$$
\sup _{x \geq 0}|\Delta f(x)| \leq \delta\left(x_{0}\right)= \begin{cases}\frac{1-q^{m}}{n p} & \text { if } x_{0}=0 \\ \min \left\{\frac{1-p^{m}}{x_{0} q}, \frac{1-p^{m+1} q^{m+1}}{(m+1) p q}\right\} & \text { if } x_{0}>0 .\end{cases}
$$

\section{Result}

The following theorem presents the main result of this study.

Theorem 3.1. For $x_{0} \in\{0, \ldots, m\}$, then we have the following:

$$
d_{x_{0}}\left(\mathcal{S}_{n}, \mathcal{B}_{m, p}\right) \leq \delta\left(x_{0}\right) \sum_{i=1}^{n}\left\{\left|p \beta_{i}-q \alpha_{i}\right|+\frac{\left(n_{i}-1\right) \beta_{i}}{\alpha_{i}+\beta_{i}+1}\right\} \frac{n_{i} \alpha_{i}}{\left(\alpha_{i}+\beta_{i}\right)^{2}}
$$

where $\delta\left(x_{0}\right)$ is defined in (2.4).

Proof. From (2.3), it follows that

$$
\begin{aligned}
d_{T V}\left(\mathcal{S}_{n}, \mathcal{B}_{m, p}\right) & =\left|E\left[\left(m-\mathcal{S}_{n}\right) p f\left(\mathcal{S}_{n}+1\right)-q \mathcal{S}_{n} f\left(\mathcal{S}_{n}\right)\right]\right| \\
& =\left|E\left[m p f\left(\mathcal{S}_{n}+1\right)-p \mathcal{S}_{n} \Delta f\left(\mathcal{S}_{n}\right)-\mathcal{S}_{n} f\left(\mathcal{S}_{n}\right)\right]\right| \\
& =\left|E\left[m p \Delta f\left(\mathcal{S}_{n}\right)\right]-p E\left[\mathcal{S}_{n} \Delta f\left(\mathcal{S}_{n}\right)\right]-\operatorname{Cov}\left(\mathcal{S}_{n}, f\left(\mathcal{S}_{n}\right)\right)\right| \\
& =\left|\sum_{i=1}^{n}\left\{E\left[\mu_{i} \Delta f\left(\mathcal{S}_{n}\right)\right]-p E\left[X_{i} \Delta f\left(\mathcal{S}_{n}\right)\right]-\operatorname{Cov}\left(X_{i}, f\left(\mathcal{S}_{n}\right)\right)\right\}\right| .
\end{aligned}
$$


Using (2.2) and Lemma 2.1, we have

$$
\begin{aligned}
d_{T V}\left(\mathcal{S}_{n}, \mathcal{B}_{m, p}\right) & =\left|\sum_{i=1}^{n}\left\{E\left[\left(\mu_{i}-p X_{i}\right) \Delta f\left(\mathcal{S}_{n}\right)\right]-\sigma_{i}^{2} E\left[w_{i}\left(X_{i}\right) \Delta f\left(\mathcal{S}_{n}\right)\right]\right\}\right| \\
& \leq \sum_{i=1}^{n} E\left\{\left|\mu_{i}-p X_{i}-\sigma_{i}^{2} w_{i}\left(X_{i}\right)\right|\left|\Delta f\left(\mathcal{S}_{n}\right)\right|\right\} \\
& \leq \sup _{x \geq 0}|\Delta f(x)| \sum_{i=1}^{n} E\left\{\left|\frac{n_{i} \alpha_{i}}{\alpha_{i}+\beta_{i}}-p X_{i}-\frac{\left(n_{i}-X_{i}\right)\left(\alpha_{i}+X_{i}\right)}{\alpha_{i}+\beta_{i}}\right|\right\} \\
& =\sup _{x \geq 0}|\Delta f(x)| \sum_{i=1}^{n} E\left\{\left|-p X_{i}-\frac{\left(n_{i}-X_{i}\right) X_{i}}{\alpha_{i}+\beta_{i}}+\frac{\alpha_{i} X_{i}}{\alpha_{i}+\beta_{i}}\right|\right\} \\
& \leq \sup _{x \geq 0}|\Delta f(x)| \sum_{i=1}^{n}\left\{\left|p \beta_{i}-p \alpha_{i}\right| E\left(X_{i}\right)+\frac{E\left(n_{i} X_{i}-X_{i}^{2}\right)}{1+\alpha_{i}+\beta_{i}}\right\} \frac{1}{\alpha_{i}+\beta_{i}} \\
& =\sup _{x \geq 0}|\Delta f(x)| \sum_{i=1}^{n}\left\{\left|p \beta_{i}-p \alpha_{i}\right|+\frac{\left(n_{i}-1\right) \beta_{i}}{1+\alpha_{i}+\beta_{i}}\right\} \frac{n_{i} \alpha_{i}}{\left(\alpha_{i}+\beta_{i}\right)^{2}} .
\end{aligned}
$$

Hence, by (2.4), (3.1) is obtained.

\section{Conclusion}

In this study, a non-uniform bound for the point metric between the distribution of a sum of $n$ independent beta binomial random variables and a binomial distribution with parameters $m=\sum_{i=1}^{n} n_{i}$ and $p=\frac{1}{m} \sum_{i=1}^{n} \frac{n_{i} \alpha_{i}}{\alpha_{i}+\beta_{i}}$ was derived by Stein's method and the beta binomial w-functions. By comparing, the bound in this study is sharper than that presented in (1.2).

\section{References}

[1] A.D. Barbour, L. Holst, S. Janson, Poisson approximation, Oxford Studies in Probability 2, Clarendon Press, Oxford, 1992.

[2] T. Cacoullos, V. Papathanasiou, Characterization of distributions by variance bounds, Statist. Probab. Lett., 7 (1989), 351-356. http://dx.doi.org/10.1016/0167-7152(89)90050-3

[3] K. Teerapabolarn, A bound on the binomial approximation to the beta binomial distribution, Int. Math. Forum, 3 (2008), 1355-1358.

[4] K. Teerapabolarn, Binomial approximation for a sum of independent beta binomial random variables, Appl. Math. Sci., 8(2014), 8929-8932. http://dx.doi.org/10.12988/ams.2014.410837 
[5] K. Teerapabolarn, P. Wongkasem, On pointwise binomial approximation by w-functions, Int. J. Pure Appl. Math., 71 (2011), 57-66.

Received: December 3, 2014; Published: January 7, 2015 